\title{
Combustion Performance and Heat Transfer in CH4/O2 Model Rocket Combustors
}

\author{
Oskar J. Haidn \\ Technische University Munchen \\ Germany
}

The propellant combination methane/oxygen $(\mathrm{CH} 4 / \mathrm{O} 2)$ is attractive for rocket engine applications due to a various advantages, i.e. higher specific impulse or less sensitive towards the onset of combustion instabilities than kerosene, less problematic in terms of leakage, auxiliary fluids or turbo machinery than liquid hydrogen and thus has seen increased interest by almost all space-faring nations. Nevertheless, none of these efforts has resulted up to now in a flying system which applies this propellant combination due to a still existing lack in understanding the key phenomena which dominate combustion performance, heat transfer and combustion stability in such a propulsion system.

The contribution will describe recent developments and results achieved studying combustion heat transfer in model combustors operated at conditions typical for rocket engines. A comparison of the single injector results from model combustors with square and circular cross sections reveals the expected similarities in combustion performance but also differences in heat fluxes which can be attributed to the corner effects. Furthermore, the corner vortices transport a sufficient amount of burnt gases back to the face plate which support flame holding and thus provide for a positive effect on combustion stability. Testing of a multi-injector circular cross section model combustor revealed the importance of flame holding for stable combustion and combustion performance for different combustion pressure levels and mixture ratios. In addition to the experimental results the contribution reports as well sample results of predicted combustion chamber pressures and temperature from different groups which have simulated a test case data from one of the single injector tests mentioned above. Interestingly, the results obtained show significant differences in combustion efficiency as well as wall heat loads and reveal that although a rather simple test cases combustion modelling for rocket engine applications has still ways to go. 